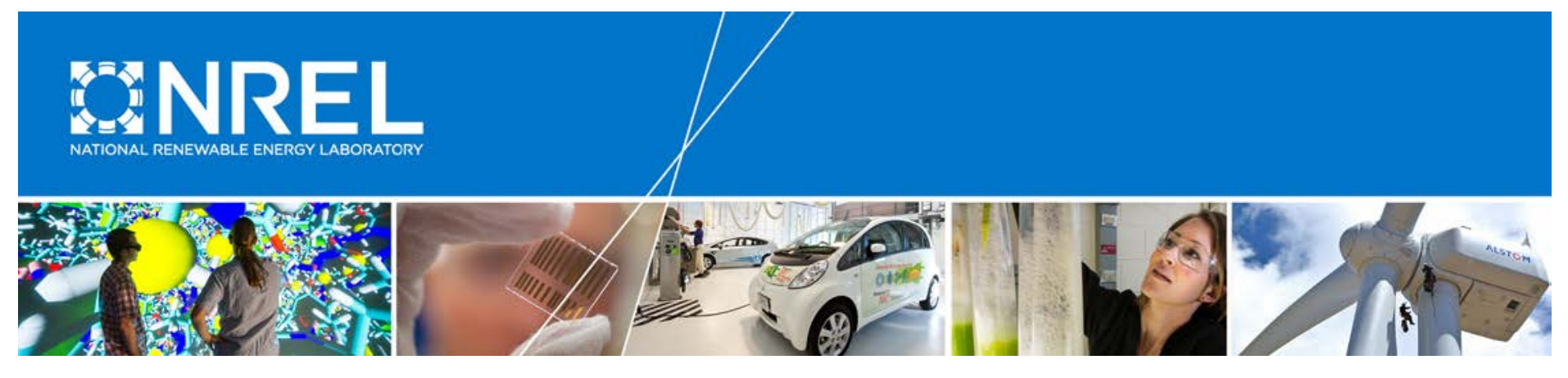

\title{
Balancing Power Absorption and Fatigue Loads in Irregular Waves for an Oscillating Surge Wave Energy Converter
}

\section{Preprint}

Nathan M. Tom, Yi-Hsiang Yu, Alan D. Wright, and Michael Lawson National Renewable Energy Laboratory

Presented at the ASME 2016 35th International Conference on Ocean, Offshore and Arctic Engineering (OMAE2016) Busan, South Korea June 19-24, 2016

NREL is a national laboratory of the U.S. Department of Energy Office of Energy Efficiency \& Renewable Energy Operated by the Alliance for Sustainable Energy, LLC

This report is available at no cost from the National Renewable Energy Laboratory (NREL) at www.nrel.gov/publications.

\section{Conference Paper}

NREL/CP-5000-65641

June 2016 


\section{NOTICE}

The submitted manuscript has been offered by an employee of the Alliance for Sustainable Energy, LLC (Alliance), a contractor of the US Government under Contract No. DE-AC36-08GO28308. Accordingly, the US Government and Alliance retain a nonexclusive royalty-free license to publish or reproduce the published form of this contribution, or allow others to do so, for US Government purposes.

This report was prepared as an account of work sponsored by an agency of the United States government. Neither the United States government nor any agency thereof, nor any of their employees, makes any warranty, express or implied, or assumes any legal liability or responsibility for the accuracy, completeness, or usefulness of any information, apparatus, product, or process disclosed, or represents that its use would not infringe privately owned rights. Reference herein to any specific commercial product, process, or service by trade name, trademark, manufacturer, or otherwise does not necessarily constitute or imply its endorsement, recommendation, or favoring by the United States government or any agency thereof. The views and opinions of authors expressed herein do not necessarily state or reflect those of the United States government or any agency thereof.

This report is available at no cost from the National Renewable Energy Laboratory (NREL) at www.nrel.gov/publications.

Available electronically at SciTech Connect http:/www.osti.gov/scitech

Available for a processing fee to U.S. Department of Energy and its contractors, in paper, from:

U.S. Department of Energy

Office of Scientific and Technical Information

P.O. Box 62

Oak Ridge, TN 37831-0062

OSTI http://www.osti.gov

Phone: 865.576.8401

Fax: 865.576.5728

Email: reports@osti.gov

Available for sale to the public, in paper, from:

U.S. Department of Commerce

National Technical Information Service

5301 Shawnee Road

Alexandria, VA 22312

NTIS http://www.ntis.gov

Phone: 800.553 .6847 or 703.605 .6000

Fax: 703.605.6900

Email: orders@ntis.gov 


\section{BALANCING POWER ABSORPTION AND FATIGUE LOADS IN IRREGULAR WAVES FOR AN OSCILLATING SURGE WAVE ENERGY CONVERTER}

\author{
Nathan M. Tom * \\ National Wind Technology Center \\ National Renewable Energy Laboratory \\ Golden, Colorado, USA \\ E-mail: Nathan.Tom@nrel.gov
}

\author{
Alan D. Wright \\ National Wind Technology Center \\ National Renewable Energy Laboratory \\ Golden, Colorado, USA \\ E-mail: Alan.Wright@nrel.gov
}

\author{
Yi-Hsiang Yu \\ National Wind Technology Center \\ National Renewable Energy Laboratory \\ Golden, Colorado, USA \\ E-mail: Yi-Hsiang.Yu@nrel.gov \\ Michael Lawson \\ National Wind Technology Center \\ National Renewable Energy Laboratory \\ Golden, Colorado, USA \\ E-mail: Michael.Lawson@nrel.gov
}

\begin{abstract}
The aim of this paper is to describe how to control the power-to-load ratio of a novel wave energy converter (WEC) in irregular waves. The novel WEC that is being developed at the National Renewable Energy Laboratory combines an oscillating surge wave energy converter (OSWEC) with control surfaces as part of the structure; however, this work only considers one fixed geometric configuration. This work extends the optimal control problem so as to not solely maximize the time-averaged power, but to also consider the power-take-off (PTO) torque and foundation forces that arise because of WEC motion. The objective function of the controller will include competing terms that force the controller to balance power capture with structural loading. Separate penalty weights were placed on the surge-foundation force and PTO torque magnitude, which allows the controller to be tuned to emphasize either power absorption or load shedding. Results of this study found that, with proper selection of penalty weights, gains in time-averaged power would exceed the gains in structural loading while minimizing the reactive power requirement.
\end{abstract}

*Address all correspondence to this author.

\section{INTRODUCTION}

Over the past year, researchers at the National Renewable Energy Laboratory have been developing a novel wave energy converter (WEC) concept that combines an oscillating surge wave energy converter (OSWEC) with active control surfaces $[1,2]$. The active control surfaces may assist in tuning the hydrodynamic properties of the device to maximize power absorption and reduce loads in larger seas to increase the operational range. The concept of controllable airfoils applied to wave energy conversion has previously been explored by Atargis Energy [3], while the concept of large-scale geometric changes has been considered in the design of Weptos [4]. However, the novel WEC considered in this paper is closer in design to a bottomfixed pitching WEC in which the main body is composed of a single large rotatable body [5]; however, increasing the number of rotatable surfaces allows for greater control over the device hydrodynamics. The development of bottom fixed OSWECs has been led by Aquamarine Power's Oyster [6], AW-Energy Oy's Waveroller [7], and Resolute Marine Energy's Surge WEC [8]; however, these designs consist of a fixed geometrical body and are generally not considered to be resonant devices [9]. 
The success of such future WEC technologies will require the development of advanced control methods that adapt device performance to maximize energy generation in operational conditions while mitigating hydrodynamic loads in extreme seas [10]. If the structural loads can be properly controlled then future WECs can be designed with thinner steel thickness to reduce overall cost. The balancing of these objectives offers an interesting design and control challenge that is beginning to garner greater research interest $[11,12]$. This multiobjective contrasts with previous works that solved the optimal control problem when focused solely on maximizing the time-averaged power (TAP). If power take-off (PTO) and structural loads are not considered in the control algorithm, then it is well known that the optimum WEC motion trajectory follows that of complex conjugate control [13], which is known to require large actuator forces and reactive power when the WEC oscillates away from the resonance frequency. The application of state-constrained optimization $[14,15]$ applied to WEC control has gained significant traction in recent years because it provides the ability to incorporate linear and nonlinear constraints. This optimization has been pursued using model predictive control [16-18] and pseudo-spectral methods $[12,19,20]$. Suboptimal strategies that eliminate reactive power, which include latching [21], declutching [22], and a nonlinear constraint on the direction of power flow [23], have been proposed yet still do not include a load metric in the optimization. It was shown in [12] that in regular waves moderate increases, roughly up to 50\%, in TAP outpaced the growth in structural loads; however, further maximization of the TAP lead to rapid growth in structural loads, thereby reducing the cost-tobenefit ratio.

This work extends the pseudo-spectral control methodology presented in [12] to irregular waves in order to determine if the same power-to-load ratios can be maintained in a more realistic sea environment. This analysis begins by introducing the hydrodynamic coefficients and mass properties of the WEC geometry used in the analysis. Next, modeling of the OSWEC timedomain pitch equation of motion is reviewed to provide the preliminaries for extension into its spectral representation. This is followed by a review of pseduo-spectral control theory, which entails the inclusion of the surge-foundation load and PTO actuator force in the optimization problem. Separate penalty weights are placed on both of the structural load contributions in an effort to provide greater control in achieving the desired performance. The effect on controller performance is observed by simulating the same sea state for combinations of penalty weights that range from maximum power absorption to minimization of structural loads. The time history of the WEC motion and PTO control torque are presented to illustrate how the increase in TAP can exceed the increase in structural loads.

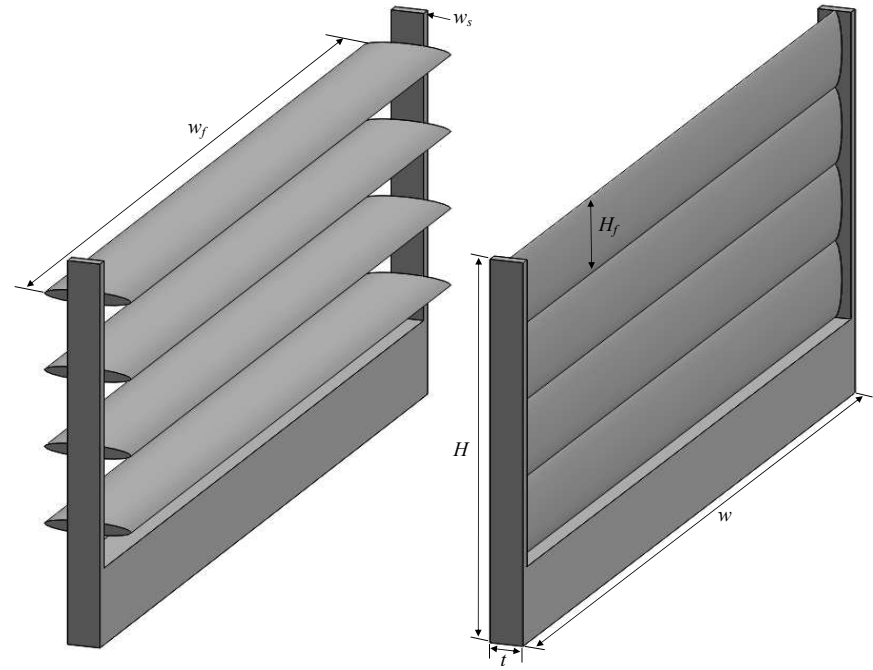

Figure 1. SOLIDWORKS RENDERING OF THE OSWEC. PERSPECTIVE VIEW OF FULLY OPEN CONFIGURATION (4 FLAPS OPEN; LEFT) AND PERSPECTIVE VIEW OF FULLY CLOSED CONFIGURATION (NO FLAPS OPEN; RIGHT).

\section{OSWEC HYDRODYNAMIC MODELING}

As outlined in previous studies $[1,2]$, the main body of the OSWEC has been replaced with a set of identical flaps that may rotate about its center axis; see Figure 1. The flaps are allowed to pitch about their center of rotation with the flap pitch angle, $\varphi$; see Figure 2. The geometric dimensions used in this study can be found in Table 1. The structural mass is assumed to be evenly distributed and the structural mass density, $\rho_{m}$, was set to half the fluid density, $\rho$. The hydrodynamic coefficients were obtained from WAMIT version 7.0 [24] at a spacing of $0.01 \mathrm{rad} / \mathrm{s}$ for wave frequencies between $0 \mathrm{rad} / \mathrm{s}-7.5 \mathrm{rad} / \mathrm{s}$. Furthermore, this work only considers the no-open-flap geometry (0-flap geometry) and the hydrodynamic coefficients can be found in Figure 3. The hydrodynamic coefficients for other flap geometries can be found in $[1,2]$ which shows over a 2 order of magnitude reduction between the geometries shown in Figure 1. A water depth of $10 \mathrm{~m}$ was chosen to calculate the hydrodynamic coefficients after reviewing previous works on bottom fixed OSWEC systems $[5,6,9]$. In [25], a study on the performance sensitivity of small surging WECs, with respect to water depth, found that a greater capture factor was obtained in shallower depths. In both [6,25] the largest water depth explored was $20 \mathrm{~m}$ while AW-Energy Oy [7] Wave Roller states that its technology can be deployed in water depths between $8-20 \mathrm{~m}$. As the water depth increases, the thickness-to-height ratio decreases and issues will begin to arise with the structural stiffness and rigid body motion may no longer be valid. Furthermore, after incorporating the additional costs associated with deployment in deeper water may likely place an upper limit of $20 \mathrm{~m}$ on the water depth. 


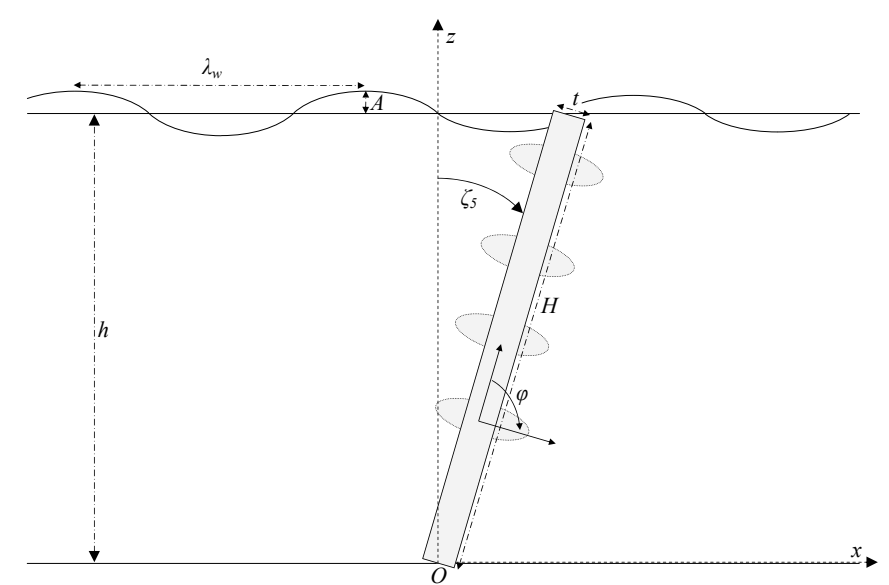

Figure 2. COORDINATE SYSTEM FOR THE DEVICE UNDER INVESTIGATION.
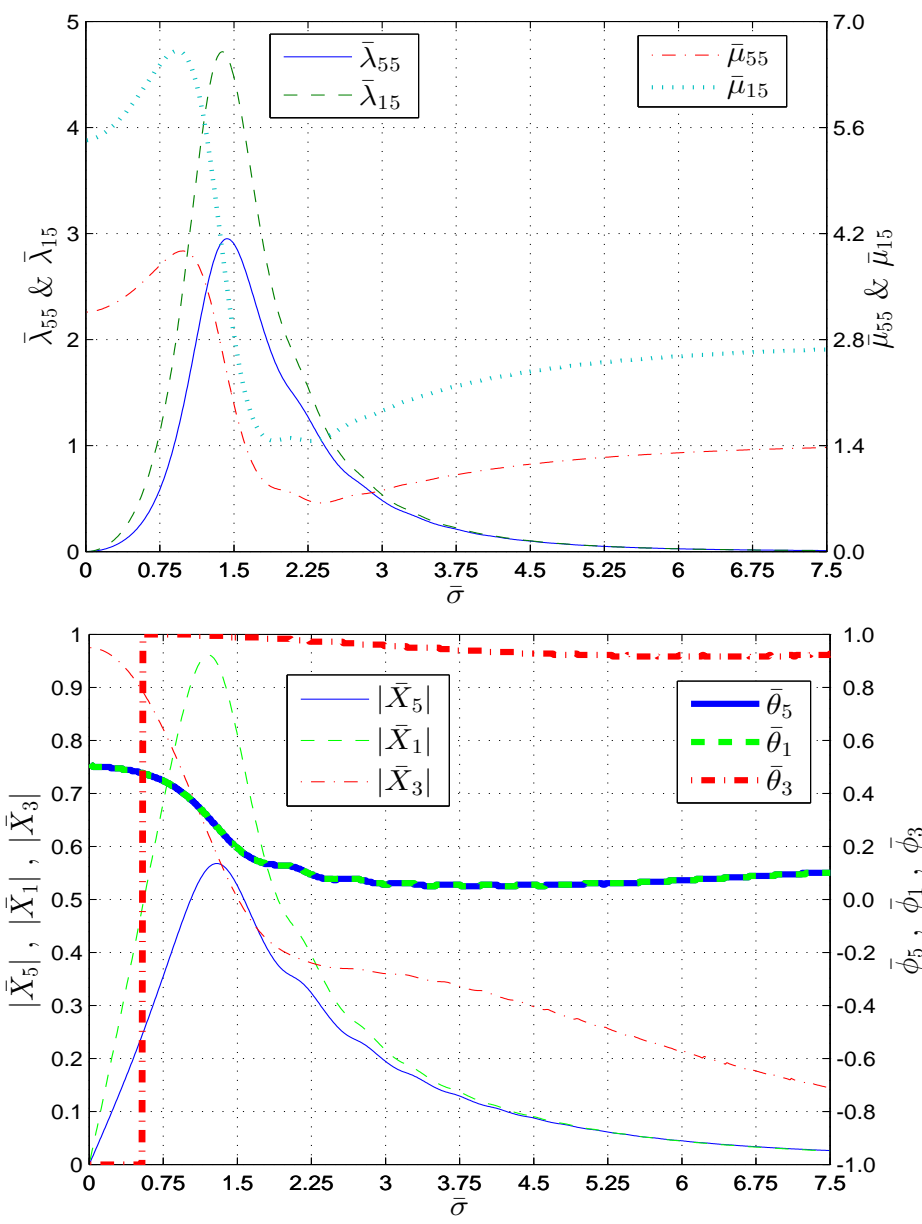

Figure 3. NONDIMENSIONAL HYDRODYNAMIC COEFFICIENTS ${ }^{1}$.
Table 1. GEOMETRIC VALUES FOR AN OSWEC.

\begin{tabular}{|c|c|}
\hline Water Depth, $h, 10 \mathrm{~m}$ & Flap Minor Axis, $t_{f}, 1 / 3 \mathrm{~m}$ \\
\hline Height, $H, 10 \mathrm{~m}$ & Flap Major Axis, $H_{f}, 2 \mathrm{~m}$ \\
\hline Thickness, $t, 3 / 4 \mathrm{~m}$ & Side Support Width, $w_{s}, 1 / 4 \mathrm{~m}$ \\
\hline Width, $w, 20 \mathrm{~m}$ & Center of Gravity, $r_{g}, 3.97 \mathrm{~m}$ \\
\hline Flap Width, $w_{f}, 19.5 \mathrm{~m}$ & Moment of Inertia, $I_{55}, 923.4 \mathrm{~kg} . \mathrm{m}^{2}$ \\
\hline Volume, $\forall, 72 \mathrm{~m}^{3}$ & Mass, $m, 36 \mathrm{t}$ \\
\hline
\end{tabular}

\section{TIME-DOMAIN PITCH EQUATION OF MOTION}

The one-degree-of-freedom time-domain pitch equation of motion is given by:

$$
I_{55} \ddot{\zeta}_{5}(t)=\tau_{e 5}(t)+\tau_{r 55}(t)+\tau_{h}(t)+\tau_{d}(t)+\tau_{m}(t)
$$

where $t$ is time, $I_{55}$ is the pitch mass moment of inertia, $\ddot{\zeta}_{5}$ is the pitch angular acceleration, $\tau_{e 5}$ is the wave-exciting pitch torque caused by the incident waves, $\tau_{r 55}$ is the wave radiation torque due to pitch motion, $\tau_{h}$ is the hydrostatic restoring torque, $\tau_{d}$ is the drag torque caused by viscous effects, and $\tau_{m}$ is the mechanical torque applied by the PTO system.

The OSWEC hydrostatic restoring moment is given by:

$$
\tau_{h}(t)=-\underbrace{\left(\rho \forall r_{b}-m r_{g}\right) g}_{C_{55}} \sin \zeta_{5}(t)
$$

where $\rho$ is the fluid density, $\forall$ is the WEC displaced volume in calm water, $r_{b}$ is the radial distance from the origin to the center of buoyancy, $m$ is the WEC mass, $r_{g}$ is the radial distance from the origin to the center of gravity, $g$ is the gravitational acceleration, and $\zeta_{5}$ is the time-varying pitch displacement. Equation (2) can be linearized by assuming small rotational motion, $\zeta_{5} \ll 1$, which allows for the following approximation to be made: $\sin \zeta_{5} \approx \zeta_{5}$.

The linear hydrodynamic wave radiation moment will be represented in the time domain using the Cummins equation [26] and is written as follows:

$$
\tau_{r 55}(t)=-\mu_{55}(\infty) \ddot{\zeta}_{5}(t)-\int_{-\infty}^{t} K_{r 55}(t-\tau) \dot{\zeta}_{5}(\tau) d \tau
$$

where $\mu_{55}(\infty)$ is the pitch-added moment of inertia at infinite frequency, and $K_{r 55}$ is the pitch radiation impulse response function, also known as the memory function because it represents the wave radiation memory effect due to past WEC motions. The relations between the time- and frequency-domain radiation co-

\footnotetext{
${ }^{1} \bar{\sigma}=\sigma \sqrt{h / g}, \bar{\mu}_{55}=\mu_{55} / \rho h^{3} w t, \bar{\lambda}_{55}=\lambda_{55} / \sigma \rho h^{3} w t, \bar{X}_{5}=X_{5} / \rho g h^{2} w, \bar{\mu}_{15}=$ $\mu_{15} / \rho h^{2} w t, \bar{\lambda}_{15}=\lambda_{15} / \sigma \rho h^{2} w t, \bar{X}_{1}=X_{1} / \rho g w h, \bar{X}_{3}=X_{3} / \rho g w t, \bar{\phi}_{i}=\phi_{i} / \pi$
} 
efficients were derived in [27]:

$$
\begin{gathered}
K_{r 55}(t)=\frac{2}{\pi} \int_{0}^{\infty} \lambda_{55}(\sigma) \cos (\sigma t) d \sigma \\
K_{r 55}(t)=-\frac{2}{\pi} \int_{0}^{\infty} \sigma\left[\mu_{55}(\sigma)-\mu_{55}(\infty)\right] \sin (\sigma t) d \sigma
\end{gathered}
$$

where $\sigma$ is the wave angular frequency, while $\mu_{55}(\sigma)$ and $\lambda_{55}(\sigma)$ are the frequency-dependent hydrodynamic radiation coefficients commonly known as the added moment of inertia and wave radiation damping.

The wave-exciting pitch torque can be written in the time domain as follows:

$$
\tau_{e 5}(t)=\int_{-\infty}^{\infty} K_{e 5}(t-\tau) \eta(\tau) d \tau
$$

where $K_{e 5}$ is the wave-excitation pitch kernel which is noncausal, and $\eta$ is the wave elevation. The relationship between the timeand frequency-domain excitation coefficients is given by:

$$
\begin{gathered}
X_{5}(\sigma)=\int_{-\infty}^{\infty} K_{e 5}(t) e^{-i \sigma t} d t \\
K_{e 5}(t)=\frac{1}{\pi} \int_{0}^{\infty}\left[\Re\left\{X_{5}(\sigma)\right\} \cos (\sigma t)-\mathfrak{I}\left\{X_{5}(\sigma)\right\} \sin (\sigma t)\right] d \sigma
\end{gathered}
$$

where $X_{5}$ is the frequency-dependent complex wave-exciting pitch-torque coefficient, $\mathfrak{R}$ is the real component, and $\mathfrak{I}$ is the imaginary component.

The final one-degree-of-freedom pitch equation of motion can now be written as:

$$
\begin{aligned}
\left(I_{55}+\mu_{55}(\infty)\right) \ddot{\zeta}_{5}(t)=- & C_{55} \zeta_{5}(t)-\int_{-\infty}^{t} K_{r 55}(t-\tau) \dot{\zeta}_{5}(\tau) d \tau \\
& +\int_{-\infty}^{\infty} K_{e 5}(t-\tau) \eta(\tau) d \tau+\tau_{m}(t)
\end{aligned}
$$

The pitch equation of motion may include a quadratic viscous drag term which has been investigated in $[1,2]$. However, the OSWEC geometry in this study has a resonance frequency outside the range of typical ocean waves and combined with small significant wave heights it will be assumed that the contribution from a quadratic viscous drag torque is minimal. A quadratic viscous drag torque can be modeled using pseudo-spectral control and the reader is directed to [28], but will not be pursued in this work and rather focus on the control concept for load reduction in irregular waves.

\section{Foundation Reaction Forces}

The structural foundation must handle the reaction forces needed to fix the WEC to the seabed. If centrifugal forces are neglected and the body remains symmetric about the vertical plane, the reaction forces in the surge, $X_{r 1}$, and heave, $X_{r 3}$, directions are given by:

$$
\begin{gathered}
A\left(X_{r 1}+X_{1}\right)=\left[-\sigma^{2} \mu_{15}+i \sigma \lambda_{15}\right] \xi_{5} \\
A\left(X_{r 3}+X_{3}\right)+(\rho \forall-m) g=0
\end{gathered}
$$

where $X_{1}$ and $X_{3}$ are the complex surge and heave wave-exciting force coefficients per unit wave amplitude, $\mu_{15}$ is the surge-pitch added mass, and $\lambda_{15}$ is the surge-pitch wave radiation damping. The following analysis will not include the static heave reaction force, $f_{m}=(\rho \forall-m) g$, in the final results because this force is unaffected by the flap pitch angle. This value is significant for ultimate strength calculation, but this work will focus on the controllable dynamic forces. The equations can be simplified and rewritten in terms of the wave-exciting force and pitch motion:

$$
\begin{gathered}
X_{r 1}=\left[-\sigma^{2} \mu_{15}+i \sigma \lambda_{15}\right] \frac{\xi_{5}}{A}-X_{1} \\
X_{r 3} \approx-X_{3}
\end{gathered}
$$

The heave reaction force is not affected by the body motion; it varies only with device geometry. The surge reaction force is affected by the OSWEC's pitch motion, which can be controlled by the PTO. The time-domain corollary of Eqn. (11) and Eqn. (12) are given by:

$$
\begin{aligned}
f_{r 1}(t) & =-\int_{-\infty}^{\infty} K_{e 1}(t-\tau) \eta(\tau) d \tau \\
& +\mu_{15}(\infty) \ddot{\zeta}_{5}(t)+\int_{-\infty}^{t} K_{r 15}(t-\tau) \dot{\zeta}_{5}(\tau) d \tau \\
f_{r 3}(t) & =-\int_{-\infty}^{\infty} K_{e 3}(t-\tau) \eta(\tau) d \tau
\end{aligned}
$$

The foundation force magnitude and angle of application is then given by:

$$
\begin{aligned}
\left|f_{r}(t)\right| & =\sqrt{f_{r 1}(t)^{2}+f_{r 3}(t)^{3}} \\
\theta_{f}(t) & \left.=\arg \left(f_{r 1}, f_{r 3}\right)\right)
\end{aligned}
$$

Notice that the time history of $\left|f_{r}(t)\right|$ will oscillate about a mean force.

\section{REVIEW OF PSEUDO-SPECTRAL CONTROL}

The discretization of the control problem is completed by approximating the velocity and PTO torque with a linear com- 
bination of basis functions [20,29]. The pitch angular velocity, $\dot{\zeta}_{5}$, and PTO control torque, $\tau_{m}$, are approximated by zero-mean truncated Fourier series with $N$ terms:

$$
\begin{aligned}
& \dot{\zeta}_{5}(t) \approx \sum_{j=1}^{N / 2} \psi_{j}^{c} \cos \left(j \sigma_{0} t\right)+\psi_{j}^{s} \sin \left(j \sigma_{0} t\right)=\Phi(t) \hat{\psi} \\
& \tau_{m}(t) \approx \sum_{j=1}^{N / 2} \tau_{j}^{c} \cos \left(j \sigma_{0} t\right)+\tau_{j}^{s} \sin \left(j \sigma_{0} t\right)=\Phi(t) \hat{\tau}
\end{aligned}
$$

where

$$
\begin{aligned}
\hat{\psi} & =\left[\psi_{1}^{c}, \psi_{1}^{s}, \ldots, \psi_{\frac{N}{2}}^{c}, \psi_{\frac{N}{2}}^{s}\right]^{\top}, \hat{\tau}=\left[\tau_{1}^{c}, \tau_{1}^{s}, \ldots, \tau_{\frac{N}{2}}^{c}, \tau_{\frac{N}{2}}^{s}\right]^{\top} \\
\Phi(t) & =\left[\phi_{1}(t), \phi_{2}(t), \ldots, \phi_{N-1}(t), \phi_{N}(t)\right] \\
& =\left[\cos \left(\sigma_{0} t\right), \sin \left(\sigma_{0} t\right), \ldots, \cos \left(\frac{N}{2} \sigma_{0} t\right), \sin \left(\frac{N}{2} \sigma_{0} t\right)\right]
\end{aligned}
$$

with the fundamental frequency given by $\sigma_{0}=2 \pi / T$ and $T$ being the chosen time duration. It can be shown that the pitch equation of motion can be described as follows:

$$
M_{55} \hat{\psi}=\hat{\tau}+\hat{e}_{5}
$$

where $\hat{e}_{5}$ represents the Fourier coefficients of the pitch waveexciting torque. The matrix $M_{55} \in \mathbb{R}^{N \times N}$ is block diagonal with the following structure:

$$
\begin{gathered}
M_{55}^{j}=\left[\begin{array}{ll}
\lambda_{55}\left(j \sigma_{0}\right) & \alpha\left(j \sigma_{0}\right) \\
-\alpha\left(j \sigma_{0}\right) & \lambda_{55}\left(j \sigma_{0}\right)
\end{array}\right], \text { for } j=1,2, \ldots, N / 2 \\
\alpha\left(j \sigma_{0}\right)=j \sigma_{0}\left(I_{55}+\mu_{55}\left(j \sigma_{0}\right)\right)-C_{55} /\left(j \sigma_{0}\right)
\end{gathered}
$$

The pitch angular velocity coefficients can then be determined explicitly from the control and pitch wave-exciting torque Fourier coefficients. This representation allows the total absorbed energy, $E$, to be written as:

$$
\begin{aligned}
E=-\int_{0}^{T} \dot{\zeta}_{5}(t) & \tau_{m}(t) d t=-\frac{T}{2} \hat{\psi}^{\top} \hat{\tau} \\
= & -\frac{T}{2}\left[\hat{\tau}^{\top}\left(M_{55}^{-1}\right)^{\top} \hat{\tau}+\hat{e}_{5}^{\top}\left(M_{55}^{-1}\right)^{\top} \hat{\tau}\right]
\end{aligned}
$$

This is in the form of a traditional quadratic problem. The symmetric component of $M_{55}^{-1}$ is positive definite [29], which guarantees that the problem is convex and the solution is globally optimal.

\section{Surge Foundation Force Penalty Term}

Load reduction will consist of limiting the forces on the WEC structure that are required to keep the point of rotation stationary. This force has two contributions that arise from the surge wave-exciting force and WEC motion. The equation for the surge foundation force can be written in a matrix form, simi- lar to Eqn. (21), as follows:

$$
\begin{gathered}
\Phi(t) \hat{f}_{r 1}=-\Phi(t) \hat{e}_{1}+\mu_{15}(\infty) \Gamma \hat{\psi}+\Phi(t)\left(G_{15}-\mu_{15}(\infty) \Gamma\right) \hat{\psi} \\
\hat{f}_{r 1}=-\hat{e}_{1}+G_{15} \hat{\psi}=-\hat{e}_{1}+G_{15} M_{55}^{-1} \hat{\tau}+G_{15} M_{55}^{-1} \hat{e}_{5}
\end{gathered}
$$

where $\hat{e}_{1}$ represents the Fourier coefficients of the surge waveexciting force, $G_{15}$ and $\Gamma$ are block matrices given in the Appendix, and Eqn. (21) has been substituted in the last expression. To maintain the convexity of the quadratic problem, the $\ell^{2}$-norm of the surge-foundation force vector was added to the objective function, which is given by:

$$
\begin{aligned}
\gamma\left|f_{r 1}\right|^{2}=\gamma \int_{0}^{T} \hat{f}_{r 1}^{\top} \Phi(t)^{\top} \Phi(t) \hat{f}_{r 1} d t=\frac{T}{2} \hat{f}_{r 1}^{\top} \hat{f}_{r 1} \\
\approx \gamma\left(2\left[\hat{e}_{1}^{\top} G_{15} M_{55}^{-1} \hat{\tau}-\hat{e}_{5}^{\top}\left(M_{55}^{-1}\right)^{\top} G_{15}^{\top} G_{15} M_{55}^{-1}\right] \hat{\tau}\right. \\
\left.-\hat{\tau}^{\top}\left(M_{55}^{-1}\right)^{\top} G_{15}^{T} G_{15} M_{55}^{-1} \hat{\tau}\right)
\end{aligned}
$$

where $\gamma$ is a penalty weight that can be used to adapt the controller performance. In the final expression for the surgefoundation force contribution, there are three constant terms independent of the control torque, which are left out of the optimization; see [12] for the full expression.

\section{Control Torque Magnitude Penalty Term}

This analysis does not consider wave-to-wave changes to the OSWEC geometry, leaving the PTO torque as the only control actuation. In an attempt to reduce computational time and large torque spikes, a penalty weight was placed on the $\ell^{2}$-norm of the PTO torque magnitude [16] as follows:

$$
\beta_{m}\left|\tau_{m}\right|^{2}=\int_{0}^{T} \beta_{m} \tau_{m}(t) \tau_{m}(t) d t=\frac{T}{2} \hat{\tau}^{\top} \beta_{m} I_{N} \hat{\tau}
$$

where $\beta_{m}$ is a penalty weight associated with the control torque magnitude and $I_{N}$ is the identity matrix of size $N$. After combining the expressions for the total absorbed power and penalty terms, the final objective function can be written as:

$$
\begin{aligned}
J= & -\frac{T}{2}\left\{-\hat{\tau}^{\top}\left[\gamma\left(M_{55}^{-1}\right)^{\top} G_{15}^{\top} G_{15} M_{55}^{-1}+\beta_{m} I_{N}-\left(M_{55}^{-1}\right)\right] \hat{\tau}\right. \\
& +\left[2 \gamma\left(\hat{e}_{1}^{\top} G_{15} M_{55}^{-1}-\hat{e}_{5}^{\top}\left(M_{55}^{-1}\right)^{\top} G_{15}^{\top} G_{15} M_{55}^{-1}\right)\right. \\
& \left.\left.+\hat{e}_{5}^{\top}\left(M_{55}^{-1}\right)^{\top}\right] \hat{\tau}\right\}
\end{aligned}
$$

\section{IRREGULAR WAVES}

The extension of psuedospectral control into the irregular wave environment will allow for evaluation of the proposed control strategy on its effectiveness for reducing fatigue loads for both WEC foundation and PTO. 


\section{Wave Spectrum Characterization}

The ocean water surface is exposed to variable winds and typically very irregular; however, the surface can be considered as a superposition of multiple regular harmonic wave components. This linear superposition principle was first introduced in hydrodynamics by St. Denis and Pierson [30], which allows an irregular wave surface to be described as:

$$
\eta(x, t)=\sum_{j=1}^{n} A_{j} \cos \left(\sigma_{j} t-k_{j} x+\varepsilon_{j}\right)
$$

where $n$ denotes the number of regular wave components used to describe the sea state, $\varepsilon$ is a random phase angle between 0 and $2 \pi$, and $k$ is the wave number. The wave number is related to the wave angular frequency through the dispersion relation. In this method the frequencies are chosen with a constant frequency step and as a result the process will repeat after a time $T_{r}$, given by $T_{r}=2 \pi / \Delta \sigma$ [31]. Unless surface elevation measurements are available, irregular seas are generally described by a wave spectrum. The wave spectrum is defined as:

$$
S^{+}(\sigma) d \sigma=\frac{1}{2} A(\sigma)^{2}
$$

For a given a wave spectrum, the wave amplitude associated for a specific wave frequency is given by:

$$
A(\sigma)=\sqrt{2 S^{+}(\sigma) d \sigma}
$$

The Bretschneider spectrum [32] was chosen for application of pseudo-spectral control and is given by:

$$
S^{+}(\sigma)=\frac{5}{16} \frac{\sigma_{p}^{4}}{\sigma^{5}} H_{s}^{2} \exp \left[-\frac{5}{4}\left(\frac{\sigma_{p}}{\sigma}\right)^{4}\right]
$$

where $\sigma_{p}$ is the modal (peak) angular frequency of the wave spectrum and $H_{s}$ is the significant wave height, traditionally defined as the mean wave height of the highest third of the waves. Relationships with the irregular wave statistics can be obtained by computing the spectral moments, $m_{n}$, of the wave spectrum:

$$
m_{n}=\int_{0}^{\infty} \sigma^{n} S^{+} d \sigma
$$

The spectral moments can be used to calculate the following quantities of the irregular wave surface elevation:

$$
H_{s}=4 \sqrt{m_{0}}, \sigma_{-1}=\frac{m_{0}}{m_{-1}}, \sigma_{1}=\frac{m_{1}}{m_{0}}, \sigma_{2}=\sqrt{\frac{m_{2}}{m_{0}}}
$$

where $H_{s}$ is the significant wave height, $\sigma_{-1}$ is the mean energy angular frequency, $\sigma_{1}$ is the mean angular frequency, and $\sigma_{2}$ is the mean zero-crossing angular frequency. The spectral moments can be calculated numerically from the available frequency data; however, the definition of the Bretschneider spectrum allows for derivation of the following analytic expressions:

$$
\left.\begin{array}{c}
m_{0}=\left(\frac{H_{s}}{4}\right)^{2} \\
m_{2}=\frac{\sqrt{5 \pi}}{2} \sigma_{p}^{2} m_{0}
\end{array}\right\} \Rightarrow \sigma_{2}=\sqrt{\frac{m_{2}}{m_{0}}}=\left(\frac{5 \pi}{4}\right)^{1 / 4} \sigma_{p}
$$

\section{Pseudo-Spectral Optimal Control in Irregular Waves}

Optimization of WEC performance in irregular waves requires a modification in calculating the Fourier coefficients of the pitch wave-exciting torque and surge wave-exciting force. The surface elevation described by Eqn. (28) will be used to calculate the wave-exciting torque as follows:

$$
\begin{aligned}
\tau_{e 5}(t)= & \Phi(t) \hat{e}_{5}=\Sigma_{j=1}^{N / 2} A\left(\sigma_{j}\right)\left[\Re\left\{X_{5}\left(\sigma_{j}\right)\right\} \cos \left(\sigma_{j} t+\varepsilon_{j}\right)(35)\right. \\
& \left.-\mathfrak{I}\left\{X_{5}\left(\sigma_{j}\right)\right\} \sin \left(\sigma_{j} t+\varepsilon_{j}\right)\right]=\Sigma_{j=1}^{N / 2} A\left(\sigma_{j}\right) \times \\
& {\left[\left(\Re\left\{X_{5}\left(\sigma_{j}\right)\right\} \cos \varepsilon_{j}-\mathfrak{I}\left\{X_{5}\left(\sigma_{j}\right)\right\} \sin \varepsilon_{j}\right) \cos \sigma_{j} t\right.} \\
& \left.-\left(\Re\left\{X_{5}\left(\sigma_{j}\right)\right\} \sin \varepsilon_{j}+\mathfrak{I}\left\{X_{5}\left(\sigma_{j}\right)\right\} \cos \varepsilon_{j}\right) \sin \sigma_{j} t\right]
\end{aligned}
$$

where the sum-difference trigonometric identities have been used in the second line. This can be put in matrix form as follows:

$$
\begin{gathered}
\Phi(t) \hat{e}_{5}=\underbrace{\left[\cos \sigma_{0} t, \sin \sigma_{0} t, \ldots, \cos \frac{N}{2} \sigma_{0} t, \sin \frac{N}{2} \sigma_{0} t\right]}_{\Phi(t)} \\
\underbrace{\left[\begin{array}{c}
A 6) \\
-A\left(\sigma_{0}\right)\left(\mathfrak{R}\left\{X_{5}\left(\sigma_{0}\right)\right\} \cos \varepsilon_{1}-\mathfrak{I}\left\{X_{5}\left(\sigma_{0}\right)\right\} \sin \varepsilon_{1}\right) \\
\vdots \\
A\left(\frac{N}{2} \sigma_{0}\right)\left(\mathfrak{R}\left\{X_{5}\left(\sigma_{0}\right)\right\} \sin \varepsilon_{1}+\mathfrak{I}\left\{X_{5}\left(\sigma_{0}\right)\right\} \cos \varepsilon_{1}\right) \\
\left.-A\left(\frac{N}{2} \sigma_{0}\right)\left(\mathfrak{R}\left\{X_{5}\left(\frac{N}{2} \sigma_{0}\right)\right\} \cos \varepsilon_{N / 2}-\mathfrak{I}\left\{X_{5}\left(\frac{N}{2} \sigma_{0}\right)\right\} \sin \varepsilon_{N / 2}\right) \mathfrak{I}\left\{X_{5}\left(\frac{N}{2} \sigma_{0}\right)\right\} \cos \varepsilon_{N / 2}\right)
\end{array}\right]}_{\hat{e}_{5}}
\end{gathered}
$$

which will have the same form for the surge and heave waveexciting force. The irregular wave-exciting Fourier coefficients can now be used in the controller routine; however, care must be taken in selecting $\sigma_{0}$ and $N$.

\section{Fatigue and Equivalent Load Calculations}

The inherent nature of irregular waves will lead to a variableamplitude cyclic time series of forces and torques. In terms of fatigue, a variable-amplitude cyclic time series may be decomposed into individual load cycles using a rainflow cycle-counting algorithm [33], and it is assumed these individual cycles may be superimposed upon one another, according to Miner's Rule. For this analysis the fatigue damage will be presented in terms of an equivalent fatigue load, which is the constant-amplitude force or torque range that would, over the same number of cycles, cause an equivalent amount of damage as the original variableamplitude stress time series [34]. The following definition will 
be used to calculate the equivalent load or torque:

$$
f^{e q}=\left(\sum_{i=1}^{C} \frac{f^{m}}{C}\right)^{1 / m}
$$

where $C$ is the cycle count and $m$ is a material property normally defined as the slope of the logarithmic S-N fatigue curve. For this analysis, $m$ will have a value of 3 , which corresponds to fatigue properties of welded steel [35].

\section{PSEUDO-SPECTRAL RESULTS}

The wave spectrum was calculated for wave frequencies between $0-7.5 \mathrm{rad} / \mathrm{s}$ with $\Delta \sigma=0.01 \mathrm{rad} / \mathrm{s}$, resulting in $T_{r}=$ $628.31 \mathrm{~s}=10.5 \mathrm{~min}$. The random phase angle seeding remained constant to maintain the same wave elevation profile per sea state. For a baseline comparison, the optimum linear damping coefficient that maximized the TAP for each sea state and geometric configuration was obtained using spectral techniques described in [2], and denoted as passive control as the PTO damping coefficient remains constant over the time series.

\section{Performance Variation with $\gamma$ and $\beta_{m}$}

The three contributions to the objective function in Eqn. (25) do not consist of the same units, and the interrelationship between them is complex. To explore the effect on controller performance, a chosen sea state was simulated using combinations of penalty weights. A sample set of results is plotted in Fig. 4, which highlights the performance of the optimization routine compared to the passive results. As to be expected, for the smallest penalty weights the capture efficiency is maximized, whereas at the largest penalty weights the minimization of the surge-foundation force and PTO control torque takes precedence and the capture efficiency is effectively zero. The intermediate range of the penalty weights provide more interesting results because a faster decrease in structural loads is observed when compared to the capture efficiency. This decrease is best viewed in the middle and bottom plots of Fig. 4, where markers have been placed for easier comparison. For each marker, the ratio of the controlled-to-passive TAP is greater than the corresponding fatigue loads. In addition, the reactive power requirement is reduced when moving from marker 1 to marker 4 , which leads to promising power-to-load ratios. The controler in this work assumes perfect efficiency of the PTO unit, though it is well known that the bidirectional efficiencies can affect results when the direction of energy flow is reversible [36].

\section{Time History of WEC Motion, Foundational Loads, and PTO Torque}

Four penalty weight combinations have been chosen to illustrate the transition from the maximum TAP to near elimination of the surge-foundation force and PTO actuator torque.
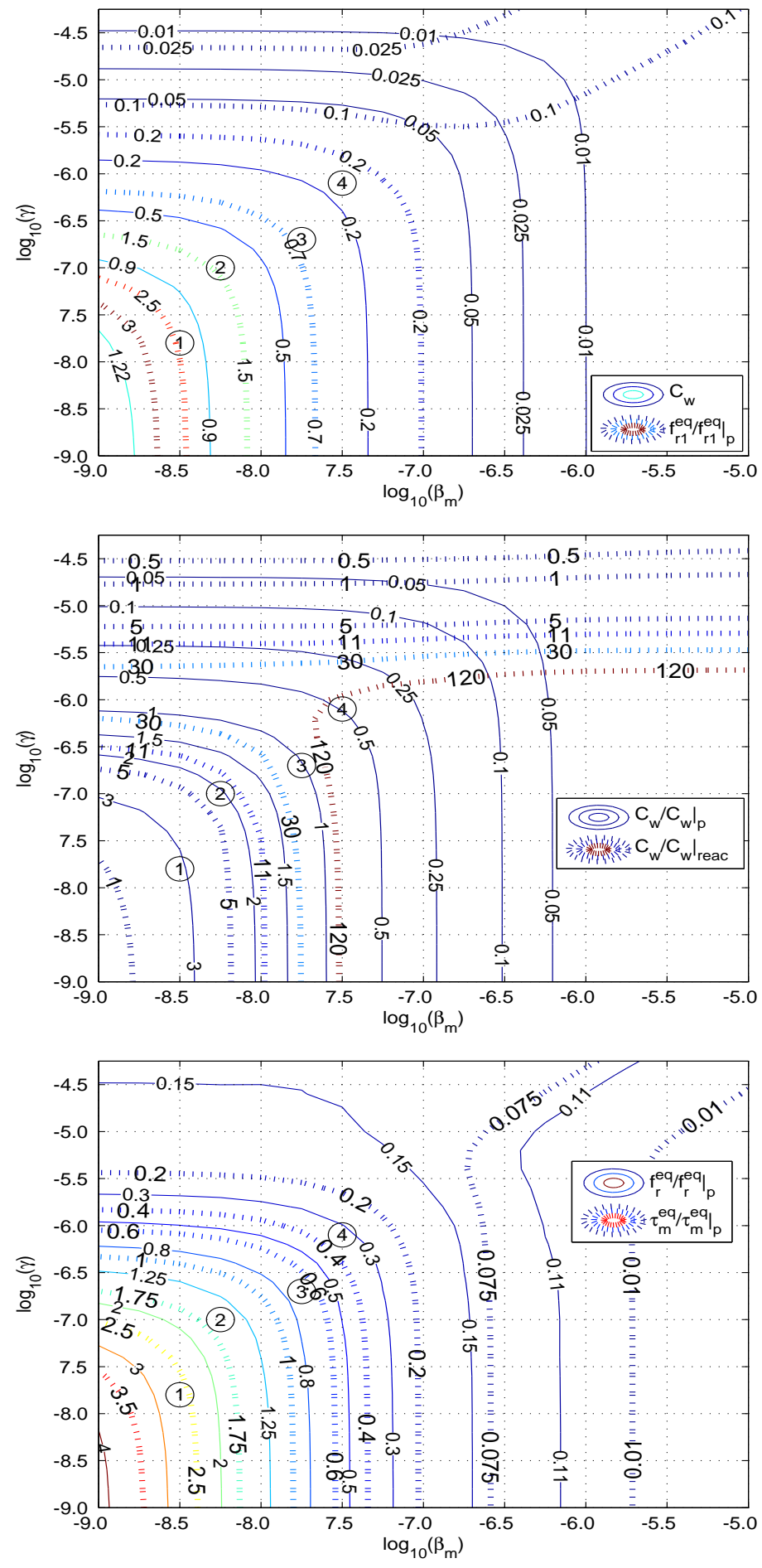

Figure 4. RESULTS FROM APPLICATION OF PSEUDO-SPECTRAL CONTROL WITH $T_{p}=10 \mathrm{~s}$ and $H_{s}=0.25 \mathrm{M}$. A PITCHDISPLACEMENT AMPLITUDE LIMIT OF $\pi / 6$ WAS USED WHILE THE NUMBER OF FOURIER COEFFICIENTS WAS SET AT $N=800$. THE PASSIVE RESULTS ARE PROVIDED AS $\left.C_{w}\right|_{p}=0.33,\left.f_{r 1}^{e q}\right|_{p}=146.1$ $\mathrm{kN},\left.f_{r}^{e q}\right|_{p}=68.7 \mathrm{kN}$, AND $\left.\tau_{m}^{e q}\right|_{p}=755.6 \mathrm{kN} \cdot \mathrm{m}$. 

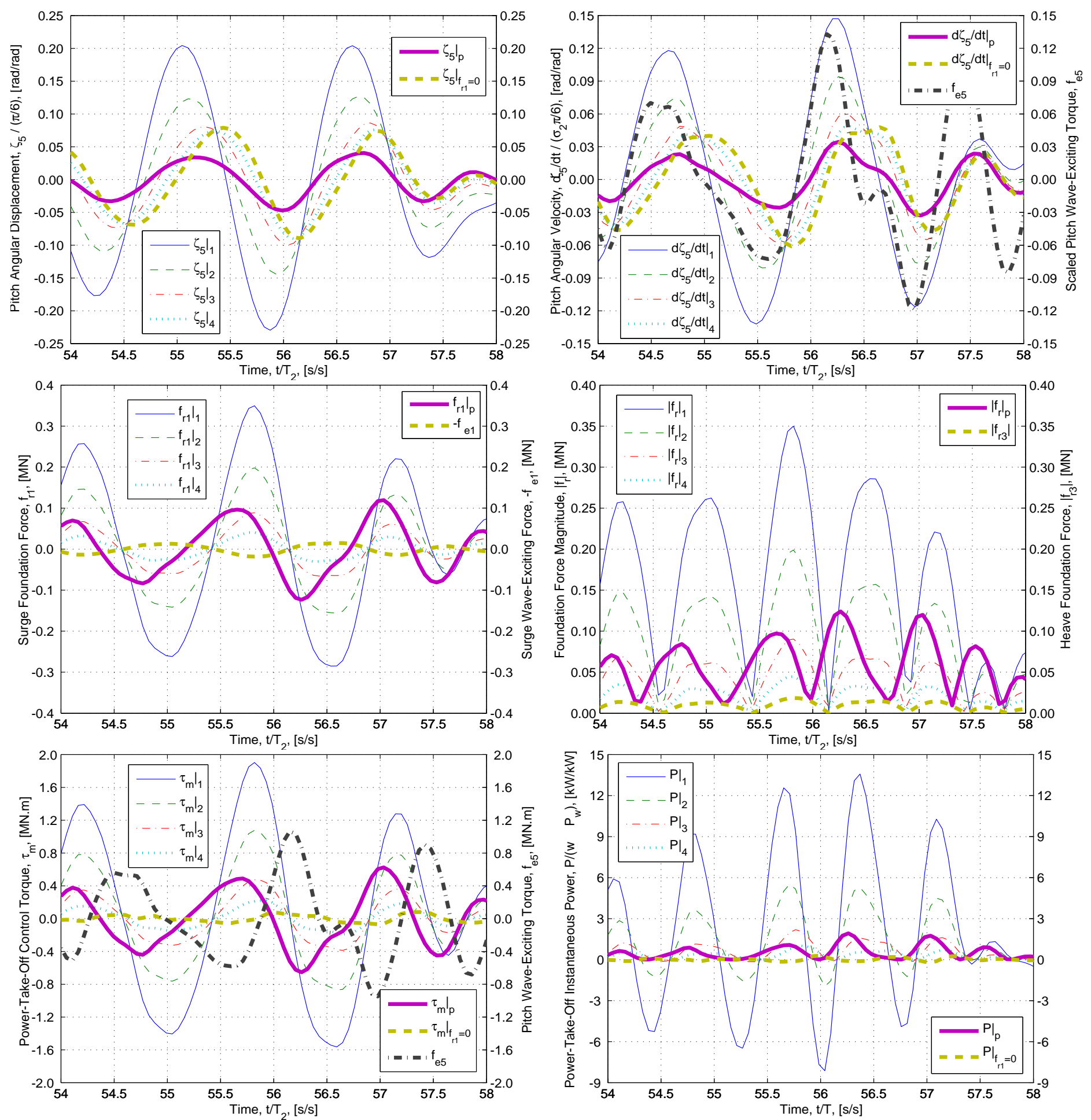

Figure 5. TIME HISTORY OF WEC MOTION, FOUNDATION FORCES, PTO CONTROL TORQUE, AND PTO POWER. RESULTS FROM APPLICATION OF PSEUDO-SPECTRAL CONTROL WITH $T_{P}=10$ SECONDS, $H_{S}=0.25$ METERS, A PITCH-DISPLACEMENT AMPLITUDE LIMIT OF $\pi / 6$ RADIANS, AND THE NUMBER OF FOURIER COEFFICIENTS WAS SET AT $N=800$. THE NUMBERS $1,2,3$, AND 4 IN THE LEGEND REFER TO THE 4 MARKERS IN THE PLOTS OF FIGURE 4. THE SUBSCRIPT $p$ DENOTES PASSIVE PERFORMANCE AS GIVEN BY [2] WHILE THE SUBSCRIPT $f_{r 1}=0$ DENOTES ELIMINATION OF THE SURGE FOUNDATION FORCE. 
These penalty weight combinations are indicated by markers in the plots of Figure 4 and the corresponding time histories are plotted in Figure 5. As the penalty weights are reduced, the controller maintains near optimum phase between the pitch angular velocity and wave-exciting torque. This condition is accompanied by the greatest PTO control torque, surge-foundation force, and reactive power. As the penalty weight is increased, the controller first maintains near optimum phase while only reducing the amplitude of motion; however, eventually a greater phase shift is allowed by the controller to eliminate a greater proportion of the surge-foundation force; refer to Eqn. (9). The larger reduction in surge-foundation force compared to the TAP is due to the phase difference between the PTO control torque and pitch angular velocity. The phase shift in pitch angular velocity assists in reducing the surge-foundation force while simultaneously moving closer in phase with the PTO control torque. If a PTO consisting of only a linear damping coefficient is used, the control torque will be in phase with the pitch angular velocity, and the reactive power component will be eliminated. Thus, for intermediate penalty weights the TAP from the controller will be near the passive result and the reactive power component is minimized. Furthermore, it can be seen that as the surge-foundation force penalty weight is increased the PTO control torque decreases. This relationship is a result of the natural body dynamics (see Figure 6) because the unforced pitch motion has nearly the same amplitude and phase needed to eliminate the surgefoundation force. Therefore, significant reductions in the surgefoundation force can be achieved by reducing the influence of the PTO torque at the expense of power production. It may be the case, however, that greater PTO actuator loads and reactive power are required to eliminate foundation loads for WECs that utilize other oscillating degrees of freedom for power production, e.g. [37].

\section{Conclusion}

This paper has used pseudo-spectral optimal control to evaluate the irregular wave performance of an OSWEC. The pitch time-domain equation of motion was constructed assuming linear hydrodynamic theory, though extension to include nonlinear effects can be made [28]. The pseudo-spectral optimal control problem was improved by including the $\ell^{2}$-norm of the surgefoundation force and PTO actuator force in the objective function. The optimizer performance was found to be adjustable based on the values chosen for the penalty weights placed on both load contributions. Verification of the optimization problem began by evaluating its performance when varying the combination of penalty weights. It was shown that as $\gamma \& \beta_{m} \rightarrow 0$ the maximum constrained power was recovered, whereas when $\gamma \& \beta_{m} \rightarrow \infty$ the surge-foundation force and PTO control torque were effectively eliminated. The results obtained in the intermediate range of penalty weights were of greater interest because they provided a better understanding of the power-to-load rela-

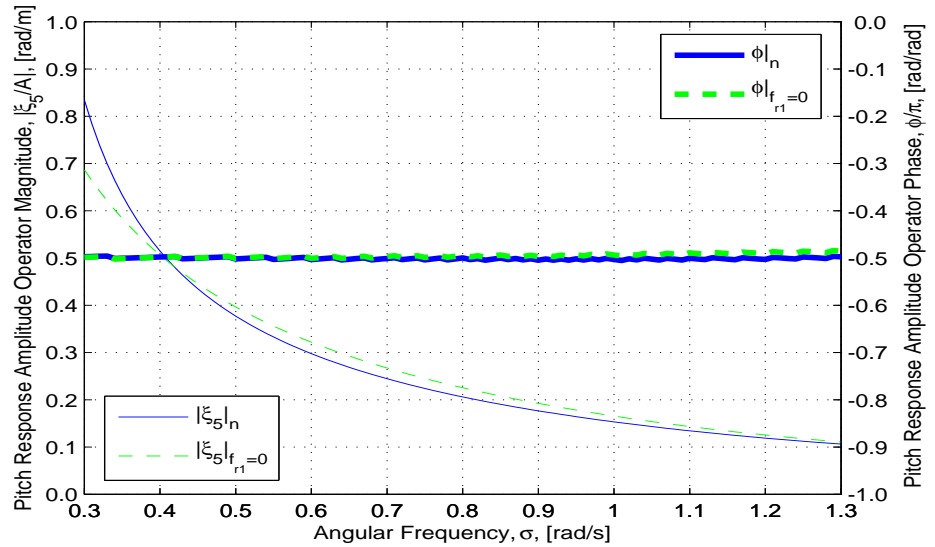

Figure 6. MAGNITUDE AND PHASE OF THE UNFORCED (NO PTO) AND ZERO SURGE-FOUNDATION FORCE RESPONSE AMPLITUDE OPERATOR. THE SUBSCRIPT ${ }_{N}$ DENOTES THE UNFORCED (NO PTO) MOTION WHILE THE SUBSCRIPT $f_{r 1}=0$ DENOTES MOTION REQUIRED FOR ELIMINATION OF SURGE-FOUNDATION FORCE.

tionships. It was found that the system loads decreased at a faster rate than the TAP, leading to favorable power-to-load ratios. As the penalty weights were increased the optimizer shifted away from optimum phase, which reduced the pitch-displacement amplitude and reactive power contribution. The reduction of the reactive power contribution is favorable because complete elimination requires nonlinear constraints on the power flow [23] that are generally slower and require iterative solvers. The pseudospectral techniques described in this paper can be solved very efficiently because of the convexity of the objective function, which is favorable for potential real-time application.

\section{ACKNOWLEDGMENT}

This work was supported by the U.S. Department of Energy under Contract No. DE-AC36-08GO28308 with the National Renewable Energy Laboratory. Funding for the work was provided by NREL's Laboratory Directed Research and Development (LDRD) Program.

\section{REFERENCES}

[1] Tom, N., Lawson, M.J., Yu, Y.H., and Wright, A.D., 2016, "Parameter study for a wave energy converter with active control surfaces. Renewable Energy (under review).

[2] Tom, N., Lawson, M.J., Yu, Y.H., and Wright, A.D., 2016, "Spectral modeling of an oscillating surge wave energy converter with control surfaces," Applied Ocean Research (accepted for publication).

[3] Siegel, S.G., Fagley, C., Romer, M., and McLaughlin, T.E., 2011, "Experimental wave cancellation using a cycloidal wave energy converter," Proceedings of the Ninth European Wave and Tidal Energy Conference (EWTEC-11), Southampton, UK, Sept. 5-9.

[4] Pecher, A., Kofoed, J.P., Larsen, T., and Marchalot, T., 2012, "Experimental study of the Weptos wave energy converter," Proceedings of the 31st International Conference on Ocean, Offshore, and Arctic Engineering (OMAE-12), Rio de Janeiro, Brazil, July 1-6. 
[5] Kurniawan, A., and Moan, T., 2012, "Characteristics of a pitching wave absorber with rotatable flap," Energy Procedia, 20, pp. 134-147.

[6] Whittaker, T., and Folley, M., 2012. Nearshore oscillating wave surge converters and the development of Oyster. Philosophical Transactions Royal Society A. 370, 345-364.

[7] Lucas, J., Livingstone, M., Vuorinen, M., and Cruz, J., 2012. Development of a wave energy converter (WEC) design tool - application to the WaveRoller WEC including validation of numerical estimates. In: Fourth International Conference on Ocean Energy, Dublin, Ireland.

[8] Ramudu, E., 2011. Ocean wave energy-driven desalination systems for off-grid coastal communities in developing countries. In: IEEE Global Humanitarian Technology Conference, Seattle, Washington, USA, pp. $1129-1142$.

[9] Gomes, R.P.F., Lopes, M.F.P., Henriques, J.C.C., Gato, L.M.C., and Falcão, A.F.O., 2015, "The dynamics and power extraction of bottomhinged plate wave energy converters in regular and irregular waves," Ocean Engineering, 96, pp. 86-99.

[10] Musial, W., Lawson, M., and Rooney, S., 2013, "Marine hydrokinetic technology (MHK) instrumentation, measurement, and computer modeling workshop," National Renewable Energy Laboratory, Technical Report NREL/TP-5000-57605.

[11] Zurkinden, A., Lambertsen, S.H., Damkilde, L., Gao, Z., and Moan T., 2013, "Fatigue Analysis of a Wave Energy Converter Taking into Account Different Control Strategies," Proceedings of the 32nd ASME International Conference on Ocean, Offshore, and Arctic Engineering (OMAE13), Nantes, France, June 9-14.

[12] Tom, N., Yu, Y. H., Lawson, M., and Wright, A. D., 2016, "Pseudospectral control of a novel oscillating surge wave energy converter in regular waves, part I: power optimization including load shedding," Ocean Engineering, (under review).

[13] Falnes, J., 2002, "Optimum control of oscillation of wave-energy converters," International Journal of Offshore and Polar Engineering, 12 (2), $147-154$.

[14] Eidsmoen, H., 1996, "Optimum control of a floating wave-energy converter with restricted amplitude," Journal of Offshore Mechanics and Arctic Engineering, 118 (2), 96-102.

[15] Hals, J., Falnes, J., Moan, T., 2011, "Constrained optimal control of a heaving buoy wave-energy converter," Journal of Offshore Mechanics and Arctic Engineering, 133 (1), 011401.

[16] Cretel, J.A.M, Lightbody, G., Thomas, G.P., and Lewis, A.W., 2011, "Maximisation of energy capture by a wave-energy point absorber using model predictive control," In: 18th World Congress of the International Federation of Automatic Control, Milano, Italy, 3714-3721.

[17] Abraham, E., Kerrigan, E.C., 2013, "Optimal active control and optimization of a wave energy converter," IEEE Transactions on Sustainable Energy, 4 (2), pp. 324-332.

[18] Li, G., Belmont, M.R., 2014, "Model predictive control of sea wave energy converters - Part I: A convex approach for the case of a single device," Renewable Energy, 69, pp. 453-463.

[19] Bacelli, G. and Ringwood, J.V., 2011,"A Control System for a Selfreacting Point Absorber Wave Energy Converter Subject to Constraints," In: 18th World Congress of the International Federation of Automatic Control, Milano, Italy, pp. 11387-11392.

[20] Herber, D.R. and Allison, J.T., 2013, Wave energy extraction maximization in irregular ocean waves using pseudospectral methods. In: ASME International Design Engineering Technical Conferences and Computers and Information in Engineering Conference, Portland, Oregon, USA.

[21] Babarit, A., Clément, A.H., 2006. Optimal latching control of a wave energy device in regular and irregular waves. Applied Ocean Research 28, 77-91.

[22] Babarit, A., Guglielmi, M., Clément, A.H., 2009, Declutching control of a wave energy converter. Ocean Engineering 36 (12), 1015-1024.
[23] Wang, L., Engström, J., Göteman, M., and Isberg, J., 2015, “Constrained optimal control of a point absorber wave energy converter with linear generator," Journal of Renewable and Sustainable Energy, 7, pp. 1-15.

[24] WAMIT Version 7.0 User Manual, http://www.wamit.com; 2013.

[25] Folley, M., Whittaker, T.J.T., and Henry, A., 2007, "The effect of water depth on the performance of a small surging wave energy converter," Ocean Engineering, 34, pp. 1265-1274.

[26] Cummins, W.E., 1962,"The impulse response function and ships motions. Schiffstechnik 9, pp. 101-109.

[27] Ogilvie, T., 1964, "Recent progress towards the understanding and prediction of ship motions," Proceedings of the Fifth Symposium on Naval Hydrodynamics, Bergen, Norway, Sept. 10-12, pp. 3-79.

[28] Bacelli, G., and Ringwood, J.V., 2014, "Nonlinear Optimal Wave Energy Converter Control with Application to a Flap-type Device," Proceedings of 19th World Congress of the International Federation of Automatic Control, Cape Town, South Africa, August 24-29, pp. 7696-7701.

[29] Bacelli, G. and Ringwood, J.V., 2015, "Numerical Optimal Control of Wave Energy Converters," IEEE Transactions on Sustainable Energy, 6 (2), pp. 133-145.

[30] St. Denis, M. and Pierson, W.J., 1953, "On the Motions of Ships in Confused Seas," Proceedings of the Annual Meeting of The Society of Naval Architects and Marine Engineers, New York, November, pp. 280-356.

[31] Baekkedal, E., 2014, "Alternative Methods of Realizing the Sea Spectrum for Time-Domain Simulations of Marine Structures in Irregular Seas," M.S. Thesis, Norwegian University of Science and Technology, Trondheim, Norway.

[32] Ochi MK, 2005, Ocean Waves: The Stochastic Approach, Cambridge University Press, Cambridge, UK, Series 6.

[33] Niesłony, A., 2009, "Determination of fragments of multiaxial service loading strongly influencing the fatigue of machine components," Journal of Renewable and Sustainable Energy, 23 (8), pp. 2712-2721.

[34] Sutherland, H.J., 1999, "On the fatigue analysis of wind turbines," Sandia National Laboratories, SAND99-0089.

[35] Ragan, P. and Manuel, L., 2007, "Comparing estimates of wind turbine fatigue loads using time-domain and spectral methods," Wind Engineering, 31 (2), pp. 83-99.

[36] Genest, R., Félicien, B., Clément, A.H., and Babarit A., 2014, "Effect of non-ideal power take-off on the energy absorption of a reactively controlled one degree of freedom wave energy converter," Applied Ocean Research, 48, pp. 236-243.

[37] Tom, N., Madhi, F., and Yeung R. W., 2016, "Balancing power absorption and structural loading for an asymmetric heave wave energy converter in regular waves," Proceedings of the 36th ASME International Conference on Ocean, Offshore, and Arctic Engineering (OMAE-16), Busan, Korea, June 19-24.

\section{A Matrix Expressions}

The time derivative of the approximated states, when using a Fourier series representation, can be written as:

$$
\dot{\zeta}_{5}=\dot{\Phi}(t) \hat{\zeta}=\Phi(t) \Gamma \hat{\zeta}
$$

where $\hat{\varsigma}$ represents the pitch angular displacement Fourier coefficients and the time-derivative matrix, $\Gamma \in \mathbb{R}^{N \times N}$, is block diagonal with the following block structure:

$$
\Gamma^{j}=\left[\begin{array}{cc}
0 & j \sigma_{0} \\
-j \sigma_{0} & 0
\end{array}\right] \text { for } j=1,2, \ldots, N / 2
$$


Using a change of variables, the surge-pitch radiation convolution integral can be represented in matrix form as follows:

$$
\begin{aligned}
\tau_{r 15}(t)=\int_{-\infty}^{t} K_{r 15}(t-\tau) \dot{\zeta}_{5} & (\tau) d \tau \\
& =\Phi(t)\left(G_{15}-\mu_{15}(\infty) \Gamma\right) \hat{\psi}
\end{aligned}
$$

where $G_{15} \in \mathbb{R}^{N \times N}$ is block diagonal with the following structure:

$$
G_{15}^{j}=\left[\begin{array}{cc}
\lambda_{15}\left(j \sigma_{0}\right) & \sigma \mu_{15}\left(j \sigma_{0}\right) \\
-j \sigma_{0} \mu_{15}\left(j \sigma_{0}\right) & \lambda_{15}\left(j \sigma_{0}\right)
\end{array}\right] \text { for } j=1,2, \ldots, N / 2
$$

